\title{
Intensidad de movimiento de escolares durante clases de educación física de colegios municipales: resultados según el profesional que efectúa las clases
}

\author{
Movement intensity of children during physical education \\ classes in public schools: results according \\ to the type of professional that teaches the class
}

\begin{abstract}
Physical education (PE) classes should contribute to increase daily energy expenditure, including moderate/vigorous activities (MVA) during $50 \%$ of the class. We determined the \% time in MVA during one PE class, identifying if there were differences according to the type of teacher in charge and the nutritional status of the children. The sample included 549 children 6 to 8 years old attending 8 public schools. Anthropometry and time in MVA were determined (using pedometers). $15.8 \%$ and $12.9 \%$ of the PE class corresponded to MVA in boys and girls respectively, with no difference by nutritional status. If the class was taught by a PE teacher, $16.9 \%$ and $14.2 \%$ corresponded to MVA; if it was taught by the classroom teacher, it was $13.7 \%$ and $10.8 \%$ in boys and girls respectively. There were differences by sex and type of teacher, but not by nutritional status. The proportion of MVA during PE classes is much lower than the recommendation of $50 \%$ of the duration of the class. The curriculum should incorporate significantly more moderate/vigorous activities and if possible classes should be taught by PE teachers.

Key Words: schoolchildren; physical education; nutritional status; movement intensity.
\end{abstract}

\section{INTRODUCCIÓN}

Están establecidos los beneficios que aporta la práctica regular de actividad física a todas las edades, ya que se asocia con mejoras en numerosos aspectos fisiológicos y psicológicos [1]. Junto con hábitos alimentarios saludables, la actividad física de cierta intensidad puede ayudar a evitar o retardar la manifestación de algunas enfermedades tales como cardiovasculares, diabetes de tipo 2, así como alteraciones osteomusculares y otras enfermedades crónicas no transmisibles.

En el contexto preventivo, la Organización Mundial de la Salud (OMS) establece como objetivo en la lucha contra la epidemia de la obesidad infantil lograr un equilibrio energético que se pueda mantener a lo largo de la vida del individuo, en donde se da énfasis a la importancia de que los niños acumulen al menos 60 minutos de actividad física diaria de intensidad moderada a vigorosa. Es importante recalcar que la actividad física debe ser de intensidad moderada a vigorosa en este grupo etario lo que implica que se debe dar mayor énfasis al aspecto aeróbico además de incorporar como mínimo tres veces por semana actividades vigorosas que refuercen en
Lorena Moreno Z. Fernando Concha $L$. Juliana Kain B.

Instituto de Nutrición y Tecnología de los Alimentos (INTA), Universidad de Chile. Santiago, Chile

Dirigir la correspondencia a: Profesora INTA, Universidad de Chile. Santiago, Chile Fono: 56-2-9781453 FAX: 56-2-2214030

E-mail: kain@inta.uchile.c.

Este trabajo fue recibido el 12 de Junio de 2012 y aceptado para ser publicado el 10 de Octubre de 2012.

particular los músculos y huesos [2].

La actividad física practicada de manera regular y programada en el niño y adolescente, favorece el desarrollo de las cualidades físico-motoras y ayuda a alcanzar un mejor nivel de aptitud física, el cual junto al factor genético es muy dependiente del estado nutricional.

Según el US Department on Health and Human Services and Department of Education [3], al mismo tiempo que aumenta la masa grasa de los niños y adolescentes, disminuye el tiempo de ejercicio realizado, dado que los niños con sobrepeso tienen más masa corporal, se puede deducir que estos realizan menos actividad física que los niños normopeso o realizan actividad física de menor intensidad [4]. En líneas generales debe aceptarse que el estado nutricional, la ingesta energética y el gasto por actividad física son determinados por numerosas influencias y restricciones que operan desde la misma infancia [5] y se reflejan tanto en la estructura corporal como en el desarrollo de hábitos conductuales.

Dado que los hábitos de actividad física y de alimentación comienzan a desarrollarse a temprana edad, los colegios tienen 
un rol fundamental en la promoción de salud y prevención de las enfermedades crónicas no transmisibles. Además las escuelas tienen varias ventajas, ya que en ellas los niños están cautivos la mayor parte del día aproximadamente durante 8 a 10 meses al año [6]. En ellas es posible pesquisar aquellos niños que están en riesgo de desarrollar alguna enfermedad crónica y así poder implementar iniciativas dentro del establecimiento o derivarlos a un centro de salud.

Dentro del currículo escolar, la clase de educación física debería ser la instancia más importante del horario escolar enfocado a mejorar los hábitos de actividad física, ya que puede influir no sólo sobre el gasto energético total, sino en el gusto por la actividad y de esa forma aumentar las probabilidades de incorporar este aspecto a la vida diaria. Diversos estudios han concluido que la clase de educación física constituye el único tiempo en el que la mitad de la población infantil realiza algún tipo de actividad física [7-8]. En el caso de los adolescentes, el grado de satisfacción con estas clases y su calidad son factores influyentes en la adherencia a la actividad física y en la adopción de un estilo de vida activo y saludable [9-11]. Ante esta responsabilidad, algunos países han optado por centrar su atención en esta asignatura, señalando directrices en beneficio de la población. Tal es el caso de Estados Unidos en donde se incluyó como objetivo de salud para la nación, metas que promueven la actividad física de intensidad moderada a vigorosa, en las clases de educación física, cuya recomendación es que se realicen diariamente con intensidad de movimiento moderada a vigorosa a lo menos durante $50 \%$ del tiempo [12].

En Chile, en los colegios públicos, de $1^{\circ}$ a $4^{\circ}$ básico, estas clases pueden ser efectuadas tanto por profesores unidocentes con y sin mención en educación física y profesores especialistas. En general el profesional mejor preparado para dirigir la asignatura de educación física es el profesor especialista quien también tiene experiencia en educación para la salud, sin embargo por falta de presupuesto, en la mayoría de colegios municipales en el primer ciclo de educación primaria, es el profesor unidocente el que efectúa esas clases. Adicionalmente, en el contexto educativo nacional es posible identificar diferencias en cuanto al número de horas dedicadas a la asignatura (dos o tres horas pedagógicas de 45 minutos cada una).

Por lo anteriormente mencionado se planteó en este estudio cumplir con los siguientes objetivos: a) determinar el tiempo de actividad física que destinan los escolares a intensidad moderada a vigorosa durante la clase de educación física b) identificar si existen diferencias en el nivel de actividad física de los niños que realizan la clase de educación física con un profesor especialista con aquellos que la efectúan con un profesor de educación general básica y c) comparar el nivel de actividad física que realizan los escolares según el estado nutricional.

\section{MÉTODOS}

Participantes: se incluyeron en este estudio los escolares de primero y segundo básico (6-8 años) de ambos sexos de los siguientes colegios municipales de la Comuna de Ñuñoa de la Región Metropolitana de Chile: Benjamín Claro Velasco, República de Costa Rica, Presidente Eduardo Frei Montalva, Brígida Walker, José Toribio Medina, Guillermo Zañartu, Lenka Franulic y República de Francia.

Se midieron al inicio 678 niños, de los cuales se excluyeron 9 debido a errores de registro y 8 porque los alumnos se retiraron antes de que finalizara la clase, dando como resultado un total de 661 niños. De ellos, 549 contaban con la evaluación nutricional, y 295 (54\%) eran varones. Este tamaño muestral sobrepasó al calculado $(n=456)$ según los resultados del único estudio que encontramos que compara los minutos de actividad moderada e intensa durante la clase, según el profesional que la ejecuta [13], considerando una diferencia de 1,5 minutos de este tipo de actividad, una desviación de 5,7 minutos, un poder del $80 \%$ y nivel de significancia del 95\%.

Mediciones: Se realizaron durante la ejecución de una clase de educación física durante los meses de octubre y noviembre 2011, en la modalidad de clases de 2 horas pedagógicas (90 minutos). Se le colocó a cada niño (14 en total por clase seleccionados al azar) un podómetro marca New Life (NL-1000), aparato que ha sido utilizado por investigadores extranjeros [14] y que ha sido validado en niños con acelerómetro [15]. Este podómetro mide los segundos y minutos en los cuales el sujeto realiza actividad moderada a vigorosa. Fue colocado al inicio de la clase a cada alumno sobre la cadera derecha afirmado por un cinturón. Se supervisó que no se manipulara el aparato por parte de los niños durante el desarrollo de la clase. Al finalizar la sesión se retiró el instrumento y se registró manualmente el tiempo de actividad moderada a vigorosa realizada. Las mediciones de peso y talla se realizaron durante el mismo período por 2 nutricionistas entrenadas. Para medir el peso se utilizó una balanza de precisión Hispana Seca modelo 804 (precisión 100 g) y para la estatura un antropómetro vertical marca Seca modelo 213 (precisión $1 \mathrm{~mm}$ ).

Análisis estadístico: el estado nutricional de los niños se evaluó utilizando el puntaje Z de IMC y la referencia OMS [16]. Se clasificaron los niños en bajo peso $(Z I M C<-1)$ normal $(Z$ $I M C>-1 \mathrm{y} \leq+1)$, sobrepeso ( $\mathrm{I} \mid M C>+1 \mathrm{y} \leq+2)$ y obeso $(\mathrm{Z}$ $I M C>+2)$. Se realizaron las siguientes comparaciones de los minutos en actividad moderada a vigorosa en la clase: según sexo, por estado nutricional y por tipo de profesor. Para la comparación del estado nutricional, se agruparon los niños en 2 categorías: bajo peso +normal $(\mathrm{N}=249$ de los cuales 6 eran bajo peso) y sobrepeso + obeso $(\mathrm{N}=300$, de los cuales 110 eran obesos). Se determinó si había diferencias significativas en los minutos totales de clases según sexo, estado nutricional y profesional, con el test de Wilcoxon debido a que la distribución de los datos no fue normal (se estableció un $p<0,05$ como nivel de significancia).

Este estudio fue aprobado por el Comité de Ética del INTA. Cada apoderado firmó el consentimiento informado en que autorizó determinar el estado nutricional y colocar el gasómetro.

\section{RESULTADOS}

La tabla 1 muestra los minutos totales en los cuales los niños están en actividad física de intensidad moderada a vigorosa durante la clase de 90 minutos según sexo y estado nutricional. Se observó que el porcentaje es muy bajo, tanto en hombres como en niñas, $15,8 \%$ y $12,9 \%$ respectivamente, sin embargo el porcentaje en los varones es significativamente mayor que en las niñas. No se observó diferencias por estado nutricional.

La tabla 2 muestra la distribución de los minutos de actividad física de intensidad moderada a vigorosa según el tipo de profesor (especialista o unidocente) que efectúa la clase, sexo y estado nutricional. Si la clase la realiza un especialista la proporción de minutos es $16,9 \%$ y $14,2 \%$ en varones y niñas respectivamente, mientras que si la realiza el unidocente, estos porcentajes son $13,7 \%$ y $10,8 \%$ respectivamente. La 
proporción es significativamente mayor en cada sexo si la clase es efectuada por el profesor de educación física $(p<0,05)$. Tampoco se observaron diferencias según el estado nutricional en relación al tipo de profesor.

\section{DISCUSIÓN}

Los resultados más importantes de este estudio muestran que durante la clase de educación física de 90 minutos, el tiempo en el cual los niños desarrollan actividad moderada a vigorosa es sumamente bajo, ya que corresponde sólo a 13 minutos, es decir, $14,4 \%$ de la clase. Los varones efectúan una mayor proporción de este tipo de actividad que las niñas, sin embargo, no se observaron diferencias según el estado nutricional. Además se observó un pequeño, aunque significativo aumento de minutos si la clase la efectuó un profesor especialista.

Estos resultados muestran lo alejados que están estas clases de cumplir la recomendación de que 50\% del tiempo los niños lo destinen a actividades de intensidad moderada a vigorosa [12].

Los datos del National Children and Youth Fitness Study, un estudio realizado con 8.800 estudiantes (niños y jóvenes)

\section{TABLA 1}

Minutos totales y porcentaje de tiempo en actividad física moderada a vigorosa durante la clase de educación física, según sexo y estado nutricional.

\begin{tabular}{lccc}
\hline & $N$ & $\begin{array}{c}\text { Minutos totales } \\
\text { act Mod/vigorosa } \\
\text { Promedio (DE) }\end{array}$ & $\begin{array}{c}\text { \% de tiempo de } \\
\text { la clase en } \\
\text { act mod/vigorosa }\end{array}$ \\
Hombres & & $14,2(5,2)$ & 15,81 \\
Total & 295 & $14,3(5,0)$ & 15,91 \\
Bajo peso + normal & 131 & $14,2(5,4)$ & 15,81 \\
Sobrepeso + obeso & 164 & $11,6(4,5)$ & 12,9 \\
Mujeres & & $12,0(4,2)$ & 13,3 \\
Total & 254 & $11,3(4,7)$ & 12,6 \\
Bajo peso + normal & 118 & & 1 \\
Sobrepeso + obesa & 136 & & \\
\hline 1= diferencia significativa por sexo $p<0,05$ & & & \\
\hline
\end{tabular}

\section{TABLA 2}

Minutos totales y porcentaje de tiempo en actividad física moderada a vigorosa durante la clase de educación física, según el tipo de profesor (especialista o unidocente) que efectúa la clase, sexo y estado nutricional.

\begin{tabular}{|c|c|c|c|}
\hline & $\mathrm{N}$ & $\begin{array}{l}\text { Minutos totales } \\
\text { act mod/vigorosa } \\
\text { Prom (DS) }\end{array}$ & $\begin{array}{l}\% \text { de tiempo de } \\
\text { la clase en } \\
\text { act mod/vigorosa }\end{array}$ \\
\hline \multicolumn{4}{|l|}{ Profesor especialista } \\
\hline Hombres total & 193 & $15,2(4,5)$ & 16,92 \\
\hline Hombres bajo peso + normal & 96 & $15,4(4,7)$ & 17,12 \\
\hline Hombres sobrepeso + obeso & 97 & $15,1(4,3)$ & 16,82 \\
\hline \multicolumn{4}{|l|}{ Profesor especialista } \\
\hline Mujeres total & 159 & $12,8(4,5)$ & 14,22 \\
\hline Mujeres bajo peso + normal & 77 & $12,9(4,3)$ & 14,32 \\
\hline Mujeres sobrepeso + obesa & 82 & $12,7(4,7)$ & 14,12 \\
\hline \multicolumn{4}{|l|}{ Profesor unidocente } \\
\hline Hombres total & 102 & $12,3(5,9)$ & 13,7 \\
\hline Hombres bajo peso + normal & 35 & $11,3(4,7)$ & 12,6 \\
\hline Hombres sobrepeso + obeso & 67 & $12,8(6,4)$ & 14,2 \\
\hline \multicolumn{4}{|l|}{ Profesor unidocente } \\
\hline Mujeres total & 95 & $9,7(3,7)$ & 10,8 \\
\hline Mujeres bajo peso + normal & 41 & $10,3(3,3)$ & 11,5 \\
\hline Mujeres sobrepeso + obesa & 54 & $9,3(3,9)$ & 10,3 \\
\hline
\end{tabular}


a nivel nacional en Estados Unidos en 1985 [17] indican que los niños obtienen de un $20 \%$ a un $40 \%$ de su actividad física en la escuela, siendo probablemente como señala Bar-Or, el único lugar en donde ellos independientemente de su proeza atlética tienen la oportunidad de participar en actividades físicas [18]. Esto demuestra la importancia que tiene la clase de educación física para crear hábitos de vida activa desde la infancia. Sin embargo, debido al limitado tiempo que dispone esta asignatura dentro del currículum escolar, la consecución de este objetivo se ve dificultada. Además, si la clase es efectuada por profesores no especializados, esto puede contribuir a que disminuya su calidad.

Estos resultados son consistentes con los obtenidos en otros estudios que concluyen que los especialistas de educación física suelen efectuar clases en las cuales la proporción de actividad física moderada a vigorosa es mayor que aquellas realizadas por maestros de aula, no sólo el tiempo dedicado difiere entre especialistas sino además el contenido del currículo. Esto fue observado en el estudio NICHD de Atención Infantil Temprana y Desarrollo de la Juventud (Study of Early Child Care and Youth Development) en 2003 en Estados Unidos, en donde las clases de educación física ejecutadas por especialistas demostraron ser lecciones más largas que proporcionaban minutos más activos haciendo que los niños gastaran más energía. Además señalaron que los profesores de educación física brindan conocimientos, prácticas y habilidades haciendo que los niños pasen menos tiempo en el juego a diferencia de los niños que tienen clases con profesores de aula [19].

El hecho de que en las clases de educación física la proporción de tiempo en actividad moderada a vigorosa es baja, ha sido observada en otros estudios. Una investigación realizada en España determinó (usando acelerómetros) que los alumnos en una clase de educación física de 120 minutos, realizaron 16.6 minutos de actividad física de intensidad moderada a vigorosa correspondiente al 9,3\% de la clase [20], un valor incluso inferior al nuestro.

En 814 niños con una edad media de 9 años que participaron en el estudio del NICHD de Atención Infantil Temprana y Desarrollo de la Juventud (Study of Early Child Care and Youth Development), se observó que en promedio, el $37 \%$ del tiempo correspondía a actividades con esa intensidad. Aún cuando los autores comentaron que este porcentaje es significativamente menor que la recomendación nacional [19] es mucho mayor que lo observado en nuestro estudio. Uno de los pocos estudios en los cuales se cumplió con la recomendación fue efectuado en las escuelas públicas de nueve distritos de EEUU en las cuales se observaron 430 clases de educación física (40 minutos cada sesión) a escolares de sexto a octavo grado. Los resultados mostraron que los niños estuvieron en promedio 16,5 minutos o $48,5 \%$ del tiempo en actividad física moderada a vigorosa, contribuyendo semanalmente con 25 minutos de actividad física vigorosa y 83 minutos de actividad física moderada a vigorosa [21].

En nuestro estudio no se observó una diferencia significativa en el tiempo destinado a actividad moderada a vigorosa según el estado nutricional, sin embargo otros estudios han mostrado que niños que presentan sobrepeso u obesidad, poseen un bajo nivel de actividad física, en comparación con sus similares que se encuentran con un estado nutricional normal $[22,23]$. Es probable que como en nuestro estudio es tan baja la proporción de tiempo en actividad moderada a vigorosa en la clase de educación física, el tiempo en ese tipo de actividad, no sea suficientemente sensible como para detectar diferencias en esa variable entre niños normopesos y con sobrepeso.

Un estudio llevado a cabo en 5 colegios de Navarra, España [4] con estudiantes de 11 y 13 años, utilizó medidas objetivas y subjetivas para medir la actividad física la que fue comparada con el estado nutricional. A través del uso de un cuestionario internacional sobre actividad física llamado IPAQ, cuyas preguntas se centraron en averiguar acerca de los tipos de actividad física en la vida cotidiana en los últimos 7 días [24], se encontró diferencias más marcadas en la actividad física vigorosa, ya que los sujetos con sobrepeso informaron practicar menos actividad física en el tiempo libre, tanto en el número de días de práctica como en la cantidad de minutos practicada en uno de esos días. En contraste, en la medición con acelerómetro, los niños con sobrepeso no mostraron un gasto calórico significativamente diferente a los niños normopeso, al igual que lo encontrado en nuestro estudio.

Otros estudios han encontrado una asociación entre el estado nutricional, la resistencia, el metabolismo basal y la fuerza. En relación a la condición física, se observó un mejor registro de VO2 máx. en el grupo normopeso que en el grupo sobrepeso y este a su vez mejor que en el grupo obesidad [25], lo cual demuestra que la condición física está relacionada de forma inversa con la cantidad de masa grasa, es decir, a mayor masa grasa menor condición física y viceversa $[26,27]$.

Los datos obtenidos en este estudio ratifican que las clases de educación física están muy por debajo de la recomendación de que un $50 \%$ de la clase sea de intensidad moderada a vigorosa. Los profesores deben dar cumplimiento al programa entregado por el Ministerio de Educación que plantea para primero y segundo básico el desarrollo de habilidades motrices básicas, el cuerpo humano y sus movimientos naturales, fortalecer el manejo del cuerpo en relación a actividades rítmicas, de recreación y de ajuste postural y, por último, valorar el cuidado del cuerpo y la higiene ambiental [28]. En este sentido, si bien se recomiendan actividades motoras lúdicas mayormente dirigidas hacia el desarrollo psicomotor del niño, no hace mención a la intensidad de las actividades que seleccione el docente. Esta es una importante limitante que presenta el área curricular; está comprobado que los estudiantes gastan más energía durante las actividades de acondicionamiento físico seguido por el juego libre [29]. Si bien es importante el desarrollo psicomotor en edades tempranas se propone que junto a este objetivo se consideren actividades físicas que otorguen beneficio a su salud de forma transversal en el programa de estudio desde los primeros niveles educativos, enfocado al desarrollo integral de los escolares, dando a conocer el sentido de cada una de las actividades favoreciendo el aprendizaje de carácter significativo, el cual puede ser mantenido con el tiempo proponiendo así un trabajo lineal en respuesta a su etapa de crecimiento.

Este estudio tiene varias limitaciones, siendo la más importante el hecho de que la medición se realizó durante una clase de educación física, por lo tanto no podría generalizarse a la calidad promedio de esas clases. Además las mediciones se efectuaron en colegios municipales de una sola comuna. Sin embargo, creemos que la calidad de esas clases en colegios municipales de otras comunas no debiera ser sustancialmente diferente.

A futuro, sería importante realizar estas mediciones en varias oportunidades en momentos donde se aborden distintos objetivos curriculares. Además, sería deseable incluir a escolares de colegios municipales de otras comunas para poder conocer si hay diferencias en el porcentaje de actividad moderada a vigorosa y tratar de explicar las razones. Es 
importante por último cuantificar cuál es la real contribución de las clases de educación física a la actividad física diaria total del niño y relacionarla con la recomendación de que ésta sea al menos de 60 minutos.

\section{CONCLUSIÓN}

Este estudio demuestra que para acercarse a la recomendación de que el $50 \%$ de la clase de educación física sea de intensidad moderada a vigorosa, se recomienda revisar los contenidos del currículum y la forma en que se están entregando con el fin de que el enfoque se oriente hacia actividades de mayor gasto energético. Además para llevar a cabo una buena enseñanza es deseable que quienes estén a cargo de la asignatura sean especialistas.

\section{RESUMEN}

Las clases de educación física debieran contribuir a aumentar el gasto energético incluyendo actividades de intensidad moderada/vigorosa (AIMI) equivalentes al 50\% de la clase. Se determinó el tiempo que destinan escolares a AIMI durante una clase de educación física, identificando si existen diferencias según el tipo de docente que efectúa la clase y el estado nutricional de los niños. La muestra incluyó 549 niños de 6-8 años de 8 escuelas municipales. Se realizaron mediciones antropométricas y tiempo en AIMI durante una clase de educación física utilizando podómetros. La proporción de clase que correspondió a AIMI fue de 15,8\% y 12,9\% en varones y niñas respectivamente, sin diferencia por estado nutricional. Si la clase fue efectuada por especialista, ésta fue $16,9 \%$ y $14,2 \%$ y por el unidocente, $13,7 \%$ y $10,8 \%$ en varones y niñas respectivamente. Se observaron diferencias por sexo y según el tipo de profesor, pero no por estado nutricional. La proporción de AIMI en la clase de educación física es sumamente baja, muy lejos de la recomendación de que sea $50 \%$ de la clase. El curriculum escolar debiera estar enfocado hacia actividades de mayor gasto energético y en lo posible quienes estén a cargo de la asignatura sean especialistas.

Palabras clave: escolares; educación física; estado nutricional; intensidad de movimiento.

\section{BIBLIOGRAFÍA}

1. Sallis J, Patrick K. Physical activity guidelines for adolescents: consensus statement, Pediatr Exerc Sci 1994; 6: 302-14.

2. World Health Organization. Global Strategy on Diet, Physical Activity and Health: Childhood what can be done, 2012. Disponible en: http://www.who.int

3. Centers for Disease Control and Prevention. Promoting better health for young people through physical activity and sports: A Report to the President from the Secretary of Health and Human Services and the Secretary of Education. Silverspring (MD): US Department on Health and Human Services and Department of Education, 2000.

4. Barquero P, Barriopedro M, Montil M. Patrones de actividad física en niños con sobrepeso y normopeso: un estudio de validez concurrente. Apunts Med l'esport 2008; 159:12734.

5. Instituto de Nutrición e Higiene de los Alimentos. Guías alimentarias para la población cubana mayores de dos años de edad, 2004. Disponible en: http://www.inha.sld.cu

6. Or Oded. La Actividad y la Aptitud Física durante la Niñez y la Adolescencia, y el Perfil de Riesgo en el Adulto. PubliCE Standard 2006; Pid:725.

7. Hernández JL, Velázquez R. La Educación Física, los estilos de vida y los adolescentes: cómo son, cómo se ven, qué saben y qué opinan. Barcelona: Grao, 2007; p.344.

8. Mackenzie T, Feldenan H.Woods S et al. Children's activity level and lessons context during third-grade physical education. Res Q Exerc Sport 1995; 66:184-93.

9. Chen A. A theoretical conceptualisation for motivation research in physical education: an integrated perspective. Quest 2001; 53:35-58.

10. Duda JL. Ejercicio físico, motivación y salud: aportaciones de la teoría de las perspectivas de meta. En: Devis J. (coord). La Educación Física, el Deporte y la Salud en el siglo XXI. Alicante: Marfil, 2001; $p$ 79-92.

11. Stelzer J, Ernest JM, Fenster MJ et al. Attitudes toward physical education: a study of high school students from four countries: Austria, Czech Republic, England, and USA. College Student J 2004; 38 (2):171-9.

12. US Department of Health and Human Services USDHHS, Estados Unidos, 2000. Disponible en: http://www.hhs. gov/

13. Oliver M, Schofield GM, Kolt GS. Physical Activity in Preschoolers Understanding Prevalence and Measurement Issues. Sports Med 2007; 37(12):1045-70.

14. McMinn D, Rowe D, Stark M, Nicol L. Validity of the New Lifestyles NL-1000 Accelerometer for Measuring Time Spent in Moderate-to-Vigorous Physical Activity in School Settings. Measurement Phys Educ Exercise Sci 2010; 14:67-78.

15. NICHD Study of Early Child Care and Youth Development Network. Frequency of activity of third-grade children in phys educ. Arch Pediatr Adolesc Med 2003; 157: 185-90.

16. World Health Organization. Measuring change in nutritional status: Guidelines for Assessing the Nutritional Impact of Suplementary Feeding Programmes for Vulnerable Groups. Ginebra, 1983.

17. Ross JG, Gilbert GG. The National Children and Youth Fitness Study: a summary of findings. J Phys Educ Recreat Dance 1985; 56: 45-50.

18. Bar-Or. A commentary to Children and fitness: a public health perspective. Res Quarterly Exercise Sports 1987; 58 (4): 304-7.

19. Nader PR. National Institute of Child Health and Human Development Study of Early Child Care and Youth Development Network. Frecuency and intensity or activity of third-grade children in physical education. Arch Pediatr Adolesc Med $2003 ;$ 157(2):185-90.

20. Martínez J, Contreras O, Lera A, Aznar S. Niveles de actividad física medida con acelerómetro en alumnos de $3^{\circ}$ ciclo de Educación Primaria: actividad diaria y sesiones de Educación Física. Rev Psicol Deporte 2011; 21(1):117-23.

21. McKenzie TL, Marshall SJ, Sallis JF, Conway TL. Student activity levels, lesson context, and teacher behaviour during middle school physical education. Res Quarterly Exercise Sport $2000 ;$ 71(3): 249-59.

22. Macmillan N. Evaluación del estado nutricional, hábitos de alimentación y actividad física en escolares de $1^{\circ}$ básico de Isla de Pascua. Rev Chil Nutr 2005; 32(3):232-7.

23. Haidar J, Abate $G$, Kogi W, Sorensen P. Risk factors for children under-nutrition with a human rights edge in rural vollages of North Wollo, Etiopía. East Afr Med 2005; 82(12):626-30.

24. Booth ML. Assessment of Physical Activity: An International Perspective. Res Quarterly Exercise Sport 2000; 71(2):11420.

25. Shriver LH, Harrist AW, Hubbs-Tait L, Topham G, Hoja M, A Barrett. Weight status, physical activity, and fitness 
among third-grade rural children. J Sch Health 2011: 81 (9):536-44.

26. Maestre JM. Connection between nutritional state and physical fitness in schoolar population. J Sport Health Res 2010; 2(2):95-108.

27. Koch E, Otarola Á, Silva C, Manríquez L, Kirschbaum A. Capacidad aeróbica, composición corporal y gasto calórico asociado a oxidación de sustratos energéticos durante el ejercicio en mujeres sedentarias con sobrepeso. Rev Chil Caardiol 2005; 24 (2): 173-83.

28. Ministerio de Educación. Programa de Estudio Educación Física, 2003.Disponible en: http://www.mineduc.cl

29. Guerrero-Fernández J. Obesidad, ¿mito o epidemia?. Mi Pediatra 2007; p. 12-5. 